\title{
Interface Cracking and Particulate Size Effect in Particle-Reinforced Metal-Matrix Composites
}

\author{
Yueguang Wei ${ }^{a}$, Tieping Li and Haiou Xie \\ State-Key Laboratory of Nonlinear Mechanics, Institute of Mechanics, Chinese Academy of \\ Sciences, Beijing 100080, China \\ aemail: Ywei@LNM.imech.ac.cn
}

Keywords: Size effect, strain gradient plasticity theory, particle-reinforced composite, interface cracking.

\begin{abstract}
The mechanical behaviors of the ceramic particle-reinforced metal matrix composites are modeled based on the conventional theory of mechanism-based strain gradient plasticity presented by Huang et al. Two cases of interface features with and without the effects of interface cracking will be analyzed, respectively. Through comparing the result based on the interface cracking model with experimental result, the effectiveness of the present model can be evaluated. Simultaneously, the length parameters included in the strain gradient plasticity theory can be obtained.
\end{abstract}

\section{Introduction}

Composites, with advantages of light weight, high strength etc have been widely applied to the planes, aerospacecrafts etc areas. Such as, the ceramic particle-reinforced metal matrix composite (PMMC) has been widely paid great attention to on its high strength.

In last about twenty years, a lot of researches on the mechanical properties of the PMMC have been done. In experimental researches, the size effect of the strength increase with particle size decrease has been reported. Simultaneously, the influences of the particle modulus, fraction etc on the composite properties have also been studied. However, these factors can not be well interpreted by using the conventional elastic-plastic theory, since in the conventional theory no any length parameters are included. Current theories used to describe the size effects are mainly, strain gradient plasticity theories, micro-polar theories and discrete dislocation theories, etc.

Nan etal[1] analyzed the mechanical properties of the particle reinforced composites using the dislocation theory from the materials sciences point of view. Wei [2] used the modified Fleck-Hutchinson strain gradient plasticity theory, cell model and finite element calculations to study the mechanical behaviors of the particle-reinforced metal matrix composite. Non-dimensional parameters for materials and cell model geometry were adopted in his analysis. He compared modeling results to experimental results of Yang etal [3] and Dai etal [4], respectively, and further predicted the corresponding length parameters included in the strain gradient plasticity theory. However, since the model adopted in his analysis didn't consider the cracking effect of the interface between particles and matrices, the calculated results had a big deviation from the experimental results, especially when applied strain was large (although both results were consistent for the small applied strain case). Xue etal [5] obtained the stress strain curves for $15 \%$ of the volume fraction of particle and for several particle sizes through finite element simulation by using a unit cell model [6] based on the mechanism-based strain gradient (MSG) plasticity theory. Nevertheless, the interface cracking was not considered in their analysis. Hu etal [7] investigated the particulate size effect in the particle-reinforced composites by using the micro-polar plasticity theory.

In the present research, the mechanical behaviors of the particle-reinforced composites will be modeled using the conventional theory of the mechanism-based strain gradient (CMSG) plasticity theory presented by Huang etal [8] and the cell model [2]. In the analysis, the two cases of considering and not considering the interface cracking between particle and matrix will be studied in detail. In the case of considering interface cracking, modeling of interface cracking process is through using a 
cohesive zone model as shown in Fig.1, by which the relationship between traction and cracking displacement can be characterized. For two cases of particle volume fraction, $15 \%$ and $50 \%$, overall stress strain relations will be modeled and investigated in detail, and the modeling results will be applied to the prediction for the experiment of $\mathrm{Al} / \mathrm{SiC}_{\mathrm{p}}$ composite and will be discussed.

\section{The CMSG Strain Gradient Plasticity Theory}

The briefly description of the CMSG theory is as follows :

$$
\begin{aligned}
& \dot{\varepsilon}_{i j}=\frac{\dot{\sigma}_{k k}}{9 K} \delta_{i j}+\frac{\dot{\sigma}_{i j}}{2 \mu}+\frac{3 \dot{\varepsilon}}{2 \sigma_{e}}\left(\frac{\sigma_{e}}{\sigma_{y} \sqrt{f^{2}\left(\varepsilon^{p}\right)+l \eta^{p}}}\right)^{m} \sigma_{i j}^{\prime} \\
& \eta^{p}=\sqrt{\frac{1}{4} \eta_{i j k}^{p} \eta_{i j k}^{p}} \\
& \eta_{i j k}^{p}=\varepsilon_{i k, j}^{p}+\varepsilon_{j k, i}^{p}-\varepsilon_{i j, k}^{p} \\
& L=18 \alpha^{2}\left(\frac{\mu}{\sigma_{y}}\right)^{2} b
\end{aligned}
$$

where $m$ is a rate-dependent index, 0 of its value will correspond to the conventional theory, in the present analysis we take $m=20 ; \eta^{p}$ is effective plastic strain gradient; $\sigma_{i j}^{\prime}$ is stress deviator; $\sigma_{e}$ is Mises effective stress; $L=l$ is material length parameter; $\alpha$ is Taylor number; $b$ is Burgers vector.

Variation equation is same form as that of conventional elastic-plastic theory, and is as follows :

$$
\int_{V} \sigma_{i j} \delta \varepsilon_{i j} d V=\int_{V} f_{i} \delta u_{i} d V+\int_{S} t_{i} \delta u_{i} d S
$$

where $f_{i}$ is volume force, $t_{i}$ is traction on the boundary $S$.

\section{The Cell Model and the Finite Element Method}

The Cell Model: Assuming that distribution of particles in the composite is periodic, the adopted cell model is shown in Fig.2.

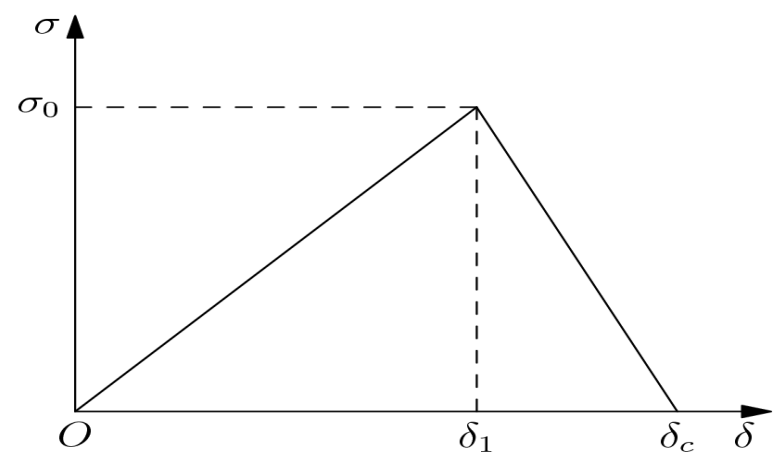

Fig.1 Cohesive zone model describing interface cracking 


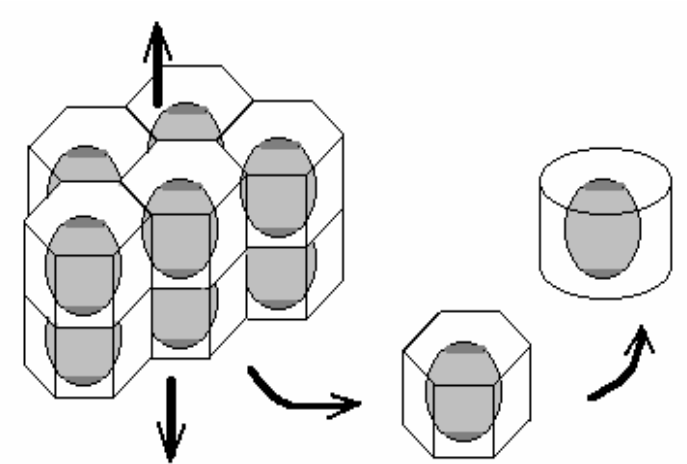

(a)

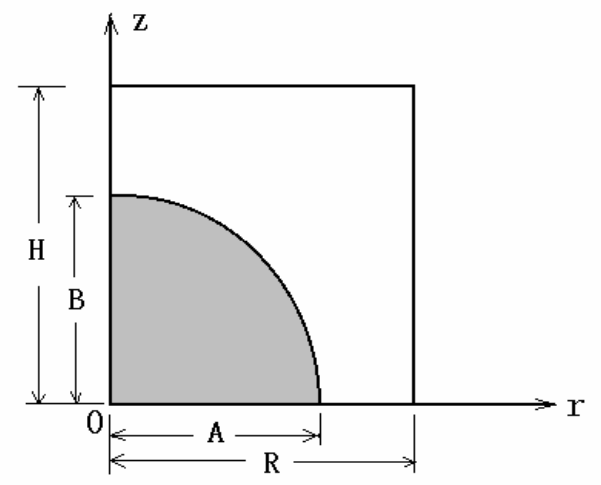

(b)

Fig.2 Cell model simplification.

From Fig.2, the cell size can be described by using three independent parameters which are the normalized particle size, aspect ratio of particle and the particle volume fraction [2].

$$
A=\left(\frac{3 k}{4 \pi}\right)^{\frac{1}{3}} \frac{V_{P}^{1 / 3}}{L} \quad, \quad B=\frac{A}{k} \quad R=\left(\frac{k}{2 \pi f_{P}}\right)^{\frac{1}{3}} \frac{V_{P}^{1 / 3}}{L} \quad, \quad H=\frac{R}{k}
$$

where $L$ is the material length parameter, $f_{p}$ is the particle volume fraction, $V_{p}$ is the particle size, $k$ is the aspect ratio of particle.

Interface Property Characterization: Interface cracking can be described by using a cohesive zone model as shown in Fig.1. Figure shows the relation between traction and relative displacement of interface cracking. In the present modeling, considering the previously published experiments about the ceramic particle-reinforced the metal matrix composites, two representative interface cases (weak adhesion and strong adhesion) will be studied:

For weak adhesion interface case with $15 \%$ of particle volume fraction,

$$
\sigma 0=\sigma \mathrm{y}, \delta 1=0.033 \mathrm{~L}, \delta \mathrm{c}=2 \mathrm{~L}
$$

For strong adhesion interface case with $50 \%$ of particle volume fraction,

$$
\sigma 0=5 \sigma \mathrm{y}, \delta 1=0.00625 \mathrm{~L}, \delta \mathrm{c}=0.2 \mathrm{~L}
$$

Material Parameters: Assuming that material of ceramic particle is homogenous, isotropic and elastic, there are two parameters, Poisson's ratio, $v_{p}$, and Young's modulus, $E_{p}$. For metal matrix, assuming that it is elastic-plastic material with strain gradient effect. Its macroscale srtess strain relation can be described by the following power-law relation:

$$
\frac{\sigma}{\sigma_{y}}=\left\{\begin{array}{cc}
\frac{E \varepsilon}{\sigma_{y}} & \varepsilon \leq \frac{\sigma_{y}}{E} \\
\left(\frac{E \varepsilon}{\sigma_{y}}\right)^{n} & \varepsilon>\frac{\sigma_{y}}{E}
\end{array}\right.
$$

where $n$ is strain hardening index, $\sigma_{y}$ is initial yield stress of material, $E$ is Young's modulus.

Boundary Conditions and Element Adopted:Considering axisymmetric condition of geometry and deformation for the cell model, the boundary conditions can be described as follows [2], 


$$
\begin{array}{clll}
u_{z}=0 & T_{r=0} & & z=0 \\
u_{z}=\text { const } & T_{r}=0 & & z=H \\
u_{r}=\text { const } & T_{z}=0 & \int_{0}^{H} T_{r} d z=0 & r=R \\
u_{r}=0 & T_{z}=0 & r=0
\end{array}
$$

The used finite element mesh is consisted of the overall mesh as shown in [2] and a magnifi- cation of local mesh at interface with the cohesive elements. Elements adopted for particle and matrix regions are the eight nodes. The induced integration scheme is used. Interface cracking process is described by using the cohesive elements.

\section{Results and Discussions}

Considering the known experimental results, in the following analysis, we take material parameters $n=0.136, E / \sigma_{y}=365, v=0.33, v_{p}=0.17, E_{p} / E=5.6$.

\begin{tabular}{|l|}
\hline S, Mises \\
(Ave. Crit. : 75\%) \\
$\begin{array}{r}+3.896 \mathrm{e}+00 \\
+3.668 \mathrm{e}+00 \\
+3.440 \mathrm{e}+00 \\
+3.211 \mathrm{e}+00 \\
+2.983 \mathrm{e}+00 \\
+2.755 \mathrm{e}+00 \\
+2.526 \mathrm{e}+00 \\
+2.298 \mathrm{e}+00 \\
+2.069 \mathrm{e}+00 \\
+1.6413 \mathrm{e}+00 \\
+1.384 \mathrm{e}+00 \\
+1.156 \mathrm{e}+00\end{array}$ \\
\hline
\end{tabular}

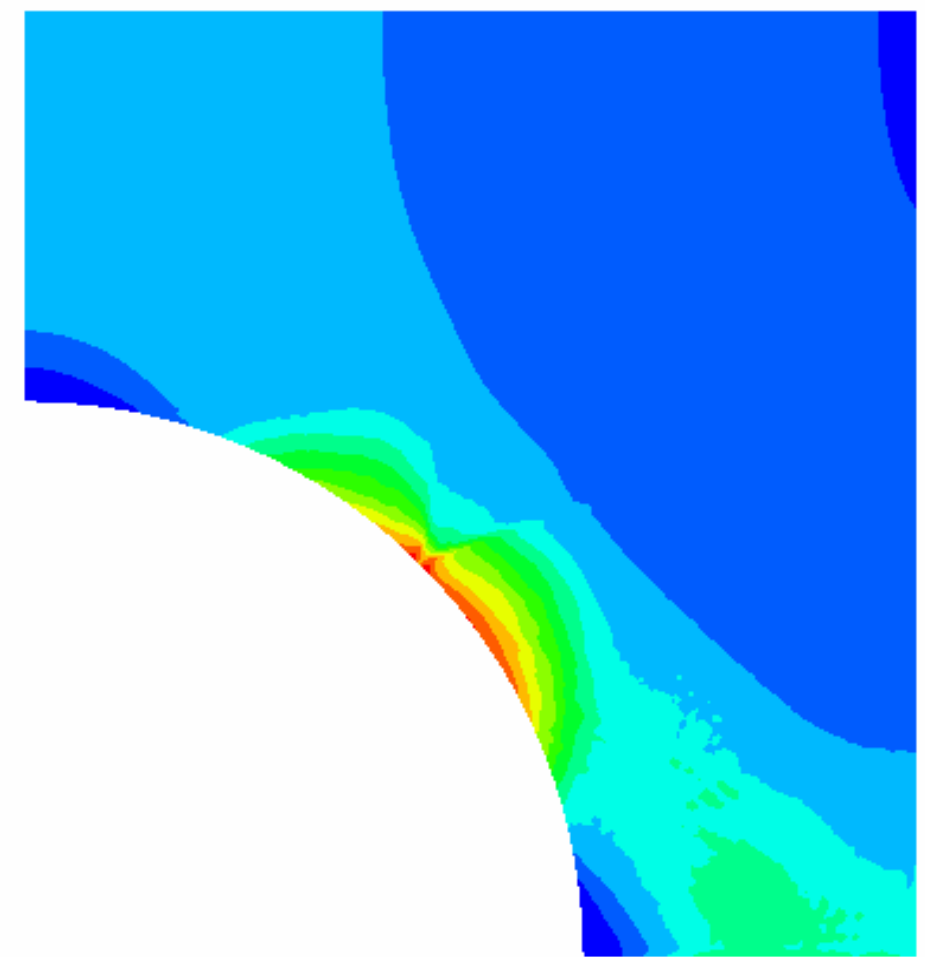

Fig.3 Mises effective stress distribution near interface for the case without interface cracking.

In our analysis, we considered a case without interface cracking first for checking the Mises effective stress distribution near interface between particle and matrix. The result is shown in Fig.3. Obviously, the maximum effective stress is always taken place at the interface, so the cracking will be occurred from there. Then in the following analysis the interface cracking cases are considered and the interface cracking process is described by using the cohesive zone model shown in Fig.1.

Fig.4(a) and Fig.4(b) show the overall stress strain curves of composites for different particle sizes, where Fig.4(a) is for $15 \%$ and Fig.4(b) is for $50 \%$ of the particle volume fractions, respectively. In these figures, the comparison of results considering interface cracking to that without interface cracking is given. From the results, the smaller the particle size, the higher the strength of composite. Through comparing the cases with and without interface cracking effects, the effect of interface 
cracking on the overall stress strain curves is obvious when strain is relatively large. Due to interface cracking effect, the stress strain curve is lower obviously.

Fig.5(a) and Fig.5(b) show comparisons of modeling results in Fig.4(a) and Fig.4(b) to the experimental results of Lloyd [9] and Yang etal[3], respectively. One can see that the modeling result considering interface cracking is consistent with the experimental one, especially for large strain case. It is to say that when applied strain is large, interface cracking is taken place, which leads to decrease of the composite strength. Simultaneously, through comparison of modeling to experimental results, one also obtains values of material length parameter included inside the strain gradient theory which is at the micron scale level, consistent with others'prediction.

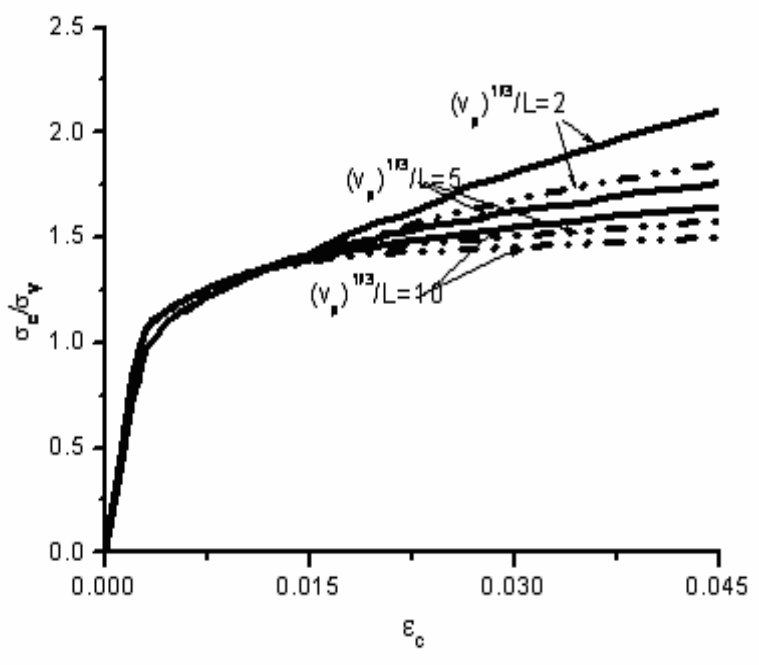

(a)

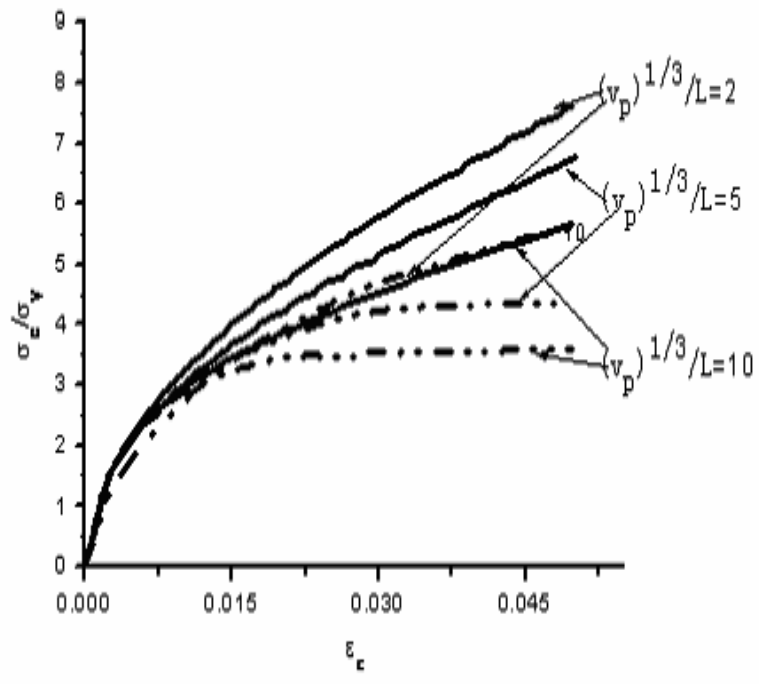

(b)

Fig.4 Overall stress strain curves for particle volume fraction 15\% (a) and 50\% (b). Dashed lines stand for the interface cracking case. Solid lines stand for no interface cracking case.

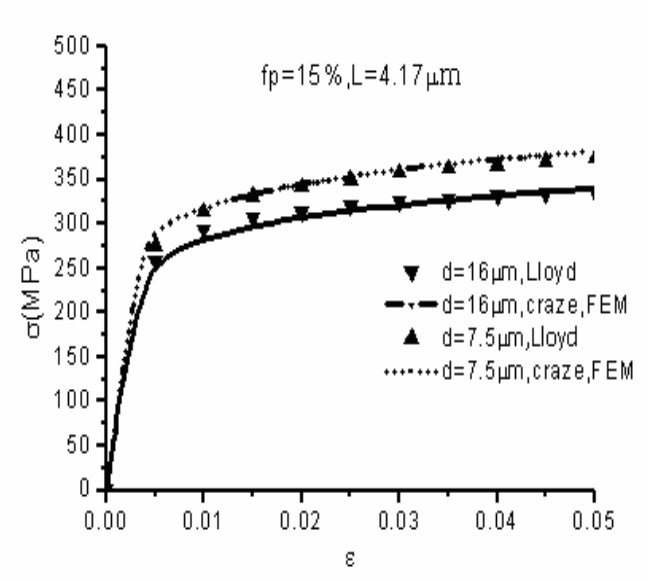

(a)

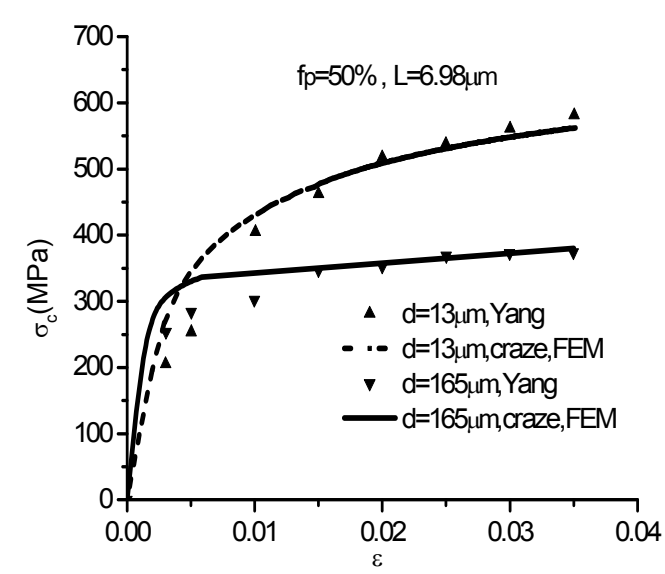

(b)

Fig.5 Comparison of predictions to experimental results of Lloyd [9] (a) and Yang etal [3] (b).

\section{Summary}

Through the present modeling, the dependences of the mechanical properties of the particle-reinforced metal matrix composites on the particle volume fraction, aspect ratio and Young's modulus, strain hardening exponent and Young's modulus of matrix are displayed. On the other hand, the dependence of the composite strength on the particle size, i.e., particle size effect is shown. 
Furthermore, the influence of cracking of interface between particle and matrix on the overall stress strain curve has been studied. The model considering the interface cracking effect gives a good description to the experimental results. Specifically, when applied strain is large, the model considering interface cracking can be still effective to describe the composite behaviors.

\section{Acknowledgments}

This work is supported by the National Science Foundation of China through grants 10428207 and 10672163, and by the Chinese Academy of Sciences through grant KJCX2-YW-M04 and by the NSFC through Innovation Project (10721202).

\section{References}

[1] C.W. NAN and D.R. Clarke: Acta Mater. Vol. 44(1996), p.3801.

[2] Y.G. Wei: Acta Mechanica Sinica Vol. 17(2001), p.45.

[3] J. Yang, C. Cady, M.S. Hu, et al: Acta Metall. Mater. Vol. 38(1990), p. 2613.

[4] L.H. Dai, Z. Ling and Y.L. Bai: Scripta Mater Vol. 41(1999), p. 245.

[5] Z. Xue, Y. Huang and M. Li: Acta Mater. Vol. 50(2002), p. 149.

[6] G. Bao and J.W. Hutchinson: Acta Metall. Mater. Vol.39(1991), p. 1871.

[7] X.N. Liu and G.K. Hu: Int. J. Plasticity Vol. 21(2005), p. 777.

[8] Y. Huang, S. Qu, K.C. Hwang, etal: Int. J. Plasticity Vol. 20(2004), p. 753.

[9] D.J. Lloyd: Int. Mater. Rev. Vol. 39(1994), p. 1. 
Advances in Fracture and Materials Behavior

10.4028/www.scientific.net/AMR.33-37

Interface Cracking and Particulate Size Effect in Particle-Reinforced Metal-Matrix Composites 10.4028/www.scientific.net/AMR.33-37.591 